\title{
Molecular Dissection of a Critical Specificity Determinant within the Amino Acid Editing Domain of Leucyl-tRNA Synthetase ${ }^{\dagger}$
}

\author{
Richard S. Mursinna, ${ }^{\ddagger}$ Keun Woo Lee,${ }^{\ddagger}$ James M. Briggs, and Susan A. Martinis* \\ Department of Biology and Biochemistry, University of Houston, Houston, Texas 77204-5001
}

Received May 29, 2003; Revised Manuscript Received October 25, 2003

\begin{abstract}
A highly conserved threonine residue marks the amino acid binding pocket within the editing active site of leucyl-tRNA synthetases (LeuRSs). It is essential to substrate specificity for the Escherichia coli enzyme in that it blocks the cognate leucine amino acid from binding in the hydrolytic editing active site. We combined mutagenesis and computational approaches to elucidate the molecular role of the critical side chain of this threonine residue. Removal of the terminal methyl group of the threonine side chain by replacement with serine yielded a mutant LeuRS that hydrolyzes Leu-tRNA ${ }^{\text {Leu }}$. Substitution of valine for the conserved threonine conferred similar activities to the wild-type enzyme. However, an additional substitution within the editing active site suggested synergistic interactions with the conserved threonine site that significantly affected amino acid editing. On the basis of our combined biochemical and computational data, we propose that the threonine 252 side chain not only sterically hinders the cognate charged leucine from binding for hydrolysis but also plays a critical role in maintaining an active site geometry that is required for the fidelity of LeuRS.
\end{abstract}

The aminoacyl-tRNA synthetases (aaRSs) ${ }^{1}$ are essential to the fidelity of protein synthesis. These enzymes covalently link a specific amino acid to its cognate tRNA in a two-step reaction (Figure 1A) $(1,2)$. First, an aminoacyl-adenylate intermediate is formed to activate the amino acid. Second, the amino acid is transferred to the $3^{\prime}$ end of the cognate tRNA. The charged tRNA product is then transported to the ribosome for protein synthesis.

Because of similarities between the side chains of the standard aliphatic amino acids (i.e., leucine, isoleucine, and valine), their cognate aaRSs have potential to bind and misactivate incorrect amino acids (3). A double sieve mechanism effectively minimizes these mistakes by employing two distinct active sites or sieves that utilize different strategies for substrate recognition (Figure 1B) (4-6). The first coarse sieve activates the cognate amino acid and also, to a lesser extent, noncognate amino acids that can fit into the amino acid binding pocket. The second fine sieve must exclude the cognate amino acid but bind misactivated and/ or mischarged amino acids for hydrolytic editing.

The catalytic core or coarse sieve that defines class I aaRSs is comprised of a Rossmann fold (7) and is responsible for amino acid activation and aminoacylation (Figure 1B) (8). Leucyl (LeuRS)-, valyl (ValRS)-, and isoleucyl (IleRS)tRNA synthetases possess a discrete polypeptide insertion, called connective polypeptide $1(\mathrm{CP} 1)$ that splits the con-

$\dagger$ This work was supported by the National Institutes of Health (GM63789), The Texas Advanced Research Program (\#003652-00161999), and The Robert A. Welch Foundation (E-1404).

* To whom correspondence should be addressed. Phone: (713) 7438390. Fax: (713) 743-8351. E-mail: smartinis@uh.edu.

$\$$ These authors contributed equally to this work.

${ }^{1}$ Abbreviations: aaRS, aminoacyl-tRNA synthetase; LeuRS, leucyltRNA synthetase; CP1, connective polypeptide 1; MD, molecular dynamics. served Rossmann fold between its third and fourth $\beta$-strands $(9,10)$. The CP1 domain folds into a discrete domain in each of these enzymes $(11-14)$ and contains a hydrolytic editing active site that operates as a fine sieve for substrate selection (Figure 1B) $(15-18)$.

Previously, we identified a conserved threonine (T252) within the CP1 domain of Escherichia coli LeuRS that is critical to amino acid specificity within the hydrolytic editing active site (17). Mutation to alanine (T252A) resulted in hydrolysis of the correctly charged Leu-tRNA ${ }^{\text {Leu }}$. Computational docking experiments using a homology modeled $E$. coli LeuRS structure (displayed in Figure 1B) suggested that the threonine residue comprises part of an amino acid binding pocket (19). Subsequent crystal structure analysis showed that this threonine side chain as well as a nearby valine (V338) could interfere with binding of the $\gamma$-branched methyl groups of leucine (20). Bulky substitutions, including tyrosine (T252Y) and phenylalanine (T252F), filled-in and blocked access to the amino acid binding pocket, thus significantly decreasing posttransfer editing activity $(18,21)$.

This critical T252 residue is highly conserved. Hence, we hypothesized that either the hydroxyl or the $\beta$-branched methyl moiety of the side chain acts as the fine discriminant to block leucine from the amino acid binding pocket (17). Since the LeuRS substrate binding pocket interacts primarily with aliphatic amino acids, it is possible that the more aliphatic valine residue would be a suitable replacement at the T252 site. Alternatively, it has also been proposed that a water molecule coordinated to the hydroxyl moiety of residue T252 is responsible for the discriminating effects of the conserved threonine (21). This suggests that serine might be a better substitute for residue T252. To test these hypotheses, we introduced a serine and valine mutation to determine if either could effectively substitute for the T252 


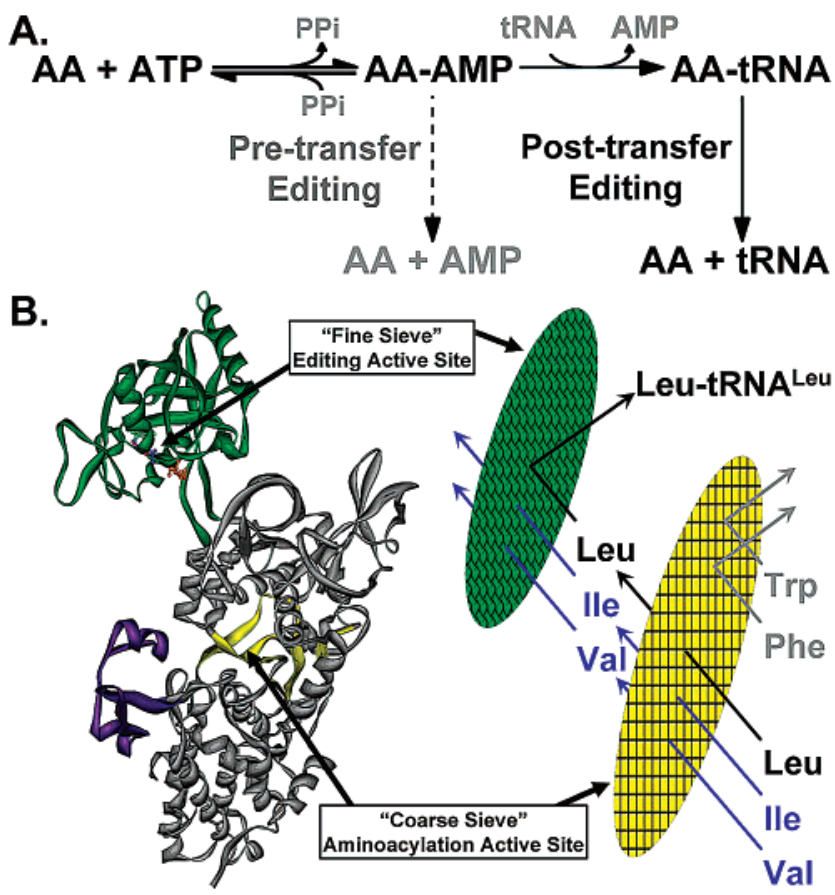

FIGURE 1: LeuRS reaction scheme, structure, and amino acid selectivity. (A) Aminoacylation and amino acid editing reaction scheme for aaRSs. Although most editing aaRSs exhibit both preand posttransfer mechanisms, E. coli LeuRS lacks pretransfer editing activity (dashed arrow). AA and PPi indicate amino acid and inorganic pyrophosphate, respectively. (B) (left) A solid ribbon diagram of the homology modeled E. coli LeuRS (19). The leucylspecific domain, $\beta$-strands comprising the Rossmann fold, and CP1 domain are colored purple, yellow, and green, respectively. (Right) depiction of the double-sieve hypothesis for LeuRS enzymes (adapted from Fersht; ref 6 ). The yellow and green filters represent the aminoacylation and editing active sites that, respectively, reside in the separate Rossmann fold and CP1 domain.

residue. Biochemical and computational characterizations of these mutant LeuRSs suggest that the T252 residue occupies a key position that not only controls amino acid specificity but also plays a critical role in establishing the geometrical positioning of other residues within the editing active site.

\section{EXPERIMENTAL PROCEDURES}

Materials. Radiolabeled amino acids were acquired from Amersham Biosciences (Piscataway, NJ). Unless otherwise noted, all other molecular biology grade reagents were either from Sigma-Aldrich, Inc. (St. Louis, MO), U.S. Biologicals (Swampscott, MA), or EM Science (Gibbstown, NJ). T7 RNA polymerase was purified as previously described (18, 22). E. coli $\mathrm{RNNA} \mathrm{UAA}_{\mathrm{UAA}}^{\mathrm{Leu}}$ was synthesized by T7 RNA polymerase runoff transcription (23) using Bst $\mathrm{N} 1$ digested ptDNAleu (18).

Wild-Type and Mutant LeuRS Preparation. Mutations were introduced into the plasmid p15EC3-1 $(18,24)$ encoding the E. coli leuS gene to generate plasmids pMURe19 and pMURe20 that express, respectively, T252S and T252V mutant LeuRSs. Complementary oligonucleotides for mutagenesis were purchased from MWG-BIOTECH Inc. (High Point, NC). The forward primers included: 5'-CCCGCCCGGACAGCTTTATGGGTTGTACC-3' for the T252S mutation and $5^{\prime}$-CCCGCCCGGACGTCTTTATGGGTTGTACC$3^{\prime}$ for the T252V mutation. T252V was also introduced into the plasmid pMURe5 (17) encoding the T248A mutant LeuRS gene to generate plasmid pMURed13 that expresses the T248A/T252V double mutant LeuRS. Polymerase chain reaction (PCR)-based mutagenesis and plasmid isolation was carried out as previously described (18). The DNA sequence of each mutant gene was confirmed by Lone Star Labs (Houston, TX). N-Terminal six-histidine-tagged wild-type and mutant proteins were expressed and purified by affinity chromatography (18).

Enzyme Assays and Characterization. Leucylation activities were measured as previously described (18). Isoleucylation and valylation assays were conducted similarly, except that $1 \mu \mathrm{M}$ enzyme was introduced and $\left[{ }^{3} \mathrm{H}\right]$-isoleucine $(600$ $\mu \mathrm{Ci} / \mathrm{mL})$ or $\left[{ }^{3} \mathrm{H}\right]$-valine $(600 \mu \mathrm{Ci} / \mathrm{mL})$ was substituted for leucine. Charged tRNA ${ }^{\text {Leu }}$ was generated and isolated as described in previous literature $(17,18)$, except that $1 \mu \mathrm{M}$ E. coli D342A/D345A double mutant LeuRS (25) was substituted to catalyze the isoleucylation and methionylation reactions. Hydrolytic editing of charged tRNA was measured in reactions that consisted of $60 \mathrm{mM}$ Tris ( $\mathrm{pH} 7.5), 10 \mathrm{mM}$ $\mathrm{MgCl}_{2}$, and either [ $\left.{ }^{3} \mathrm{H}\right]$-Leu-, $\left[{ }^{3} \mathrm{H}\right]-\mathrm{Ile}$-, or $\left[{ }^{35} \mathrm{~S}\right]$-Met-tRNA ${ }^{\text {Leu }}$ and initiated with 1-30 nM enzyme $(17,18,26)$. Kinetic rate constants were measured for aminoacylation by incorporating six different leucine and tRNA ${ }^{\text {Leu }}$ concentrations ranging from 0.5 to $19 \mu \mathrm{M}$ leucine and 0.3 to $40 \mu \mathrm{M}$ in vitro transcribed tRNA $\mathrm{UAA}_{\mathrm{UA}}^{\mathrm{Lu}}$. Kinetic rate constants were measured for hydrolytic editing by incorporating six different Leu-tRNA $A_{\text {UAA }}^{\mathrm{Leu}}$ concentrations ranging from 80 to $2000 \mathrm{nM}$ charged tRNA.

Computational Analysis. A homology-modeled structure of E. coli LeuRS (19) was previously built based on the X-ray crystal structure of $T$. thermophilus LeuRS (11). To minimize computing time, the $\mathrm{CP} 1$ domain was isolated from the fulllength protein by excision at amino acids I224 and L417. These terminal amino acids are located on the $\beta$-strands linking the CP1 domain to the main body. The site of excision retains approximately six residues of each $\beta$-strand on the CP1 domain. The newly established terminal CP1 domain residues were neutralized as $\mathrm{NH}_{2}$ and $\mathrm{COOH}$ to avoid unnaturally strong Coulombic interactions between the artificial termini. This truncated structure provided a wildtype LeuRS CP1 domain model that was mutated using the BIOPOLYMER module of the INSIGHTII program (27) to mimic mutations made biochemically.

The four prepared LeuRS CP1 domain models (wild-type, T252A, T252S, and T252V) were fully hydrated within a $64 \times 64 \times 64 \AA$ water box that contained 7620 water molecules. The total number of atoms for each system was about 25,800 . The protein structure file of the entire system was generated with CHARMM (28). Minimization and molecular dynamics (MD) simulations were carried out with CHARMM27 parameters $(28,29)$ using NAMD $(30)$, due to its demonstrated excellent performance in parallel environments. Periodic boundary conditions and a dielectric constant of 1 were used throughout the simulations. A spherical cutoff of $10.5 \AA$ was used to truncate pairwise interactions. The pair list for each atom was defined to include atoms within $11.5 \AA$. The switching function was used for distances between 9.5 and $10.5 \AA$. Energy minimizations were performed by using a conjugate gradient method consisting of 5000 steps. The temperature for each system was increased from 0 to $298 \mathrm{~K}$, then equilibrated during the first $50 \mathrm{ps}$ of $\mathrm{MD}$, and thereafter controlled by Berendsen's 
coupling method (31) using a heat bath at $298 \mathrm{~K}$ for the subsequent $1000 \mathrm{ps}$ of MD. The pressure was also managed by Berendsen's method at 1 bar with a compressibility of 0.000049 bar $^{-1}$ and a relaxation time of $500 \mathrm{fs}$. The time step of the MD simulation was 2 fs. The SHAKE algorithm was used to constrain all bonds to hydrogen atoms.

\section{RESULTS}

Mutational Analysis of the T252 Discriminant Factor. The conserved T252 residue acts as a fine discriminator in the E. coli LeuRS editing active site by blocking the correctly charged Leu-tRNA ${ }^{\text {Leu }}$ from binding and being hydrolyzed. We performed mutational analysis to better delineate the molecular role of the threonine side chain in substrate recognition and editing activity. We introduced a serine substitution (T252S) that would retain the hydroxyl moiety. However, it is less bulky due to the removal of the branched methyl moiety of the wild-type threonine residue. In contrast, a valine substitution (T252V) would maintain the bulk and shape of the threonine side chain but lacks a hydroxyl group that could be key to hydrogen bonding.

The purified wild-type, T252S, and T252V mutant LeuRS proteins were tested for cognate leucine aminoacylation activities (i.e., leucylation). Although leucylation by the T252V mutant LeuRS was similar to the wild-type enzyme, introduction of serine decreased the overall yields of charged Leu-tRNA ${ }^{\text {Leu }}$ (Figure 2A). As shown previously (17), the T252A mutant LeuRS produces significantly less charged tRNA because this mutation alters specificity and results in the hydrolysis of the correctly charged Leu-tRNA ${ }^{\text {Leu. Inter- }}$ estingly, the $k_{\text {cat }}$ of Leu-tRNA ${ }^{\text {Leu }}$ formation by the T252S mutant LeuRS is either similar or slightly increased as compared to that of the wild-type and T252V mutant LeuRSs (Table 1). Thus, both mutants can effectively aminoacylate tRNA. However, the T252S mutation lowers LeuRS accumulation levels of the charged product.

We hypothesized that the T252S mutant LeuRS could be editing Leu-tRNA ${ }^{\text {Leu }}$ in a similar manner as the T252A mutant enzyme. Thus, the editing reaction would compete with the aminoacylation reaction and lower the overall yield of correctly charged product. As Figure 2B shows, direct hydrolysis of Leu-tRNA ${ }^{\text {Leu }}$ by the T252A and T252S mutant LeuRSs were greatly stimulated relative to the wild-type and T252V mutant enzymes. The $k_{\text {cat }}$ of Leu-tRNA ${ }^{\text {Leu }}$ hydrolysis by the T252S mutant LeuRS is increased over 6-fold when compared to the wild-type LeuRS (Table 2). In contrast, the T252V mutant LeuRS appears to largely block editing of the correctly charged Leu-tRNA ${ }^{\text {Leu}}$, similar to that of the wild-type enzyme (Figure $2 \mathrm{~B})$. Although hydrolysis $\left(k_{\mathrm{cat}}\right)$ is increased for the $\mathrm{T} 252 \mathrm{~V}$ mutant LeuRS, the specificity constants $\left(k_{\text {cat }} / K_{\mathrm{M}}\right)$ are similar.

Although the T252S mutant LeuRS hydrolyzes LeutRNA $^{\text {Leu }}$, it is able to stably accumulate correctly charged product, albeit at reduced yields. If the synthesis of LeutRNA $^{\text {Leu }}$ and its subsequent hydrolysis compete under the conditions that are used in standard aminoacylation assays, one might expect that charged tRNA production would occur, but the levels of product attained would be compromised. This might be explained based on a simple comparison of the kinetic constants of each enzyme (Tables 1 and 2) where the turnover number of Leu-tRNA ${ }^{\text {Leu }}$ hydrolysis is signifi-
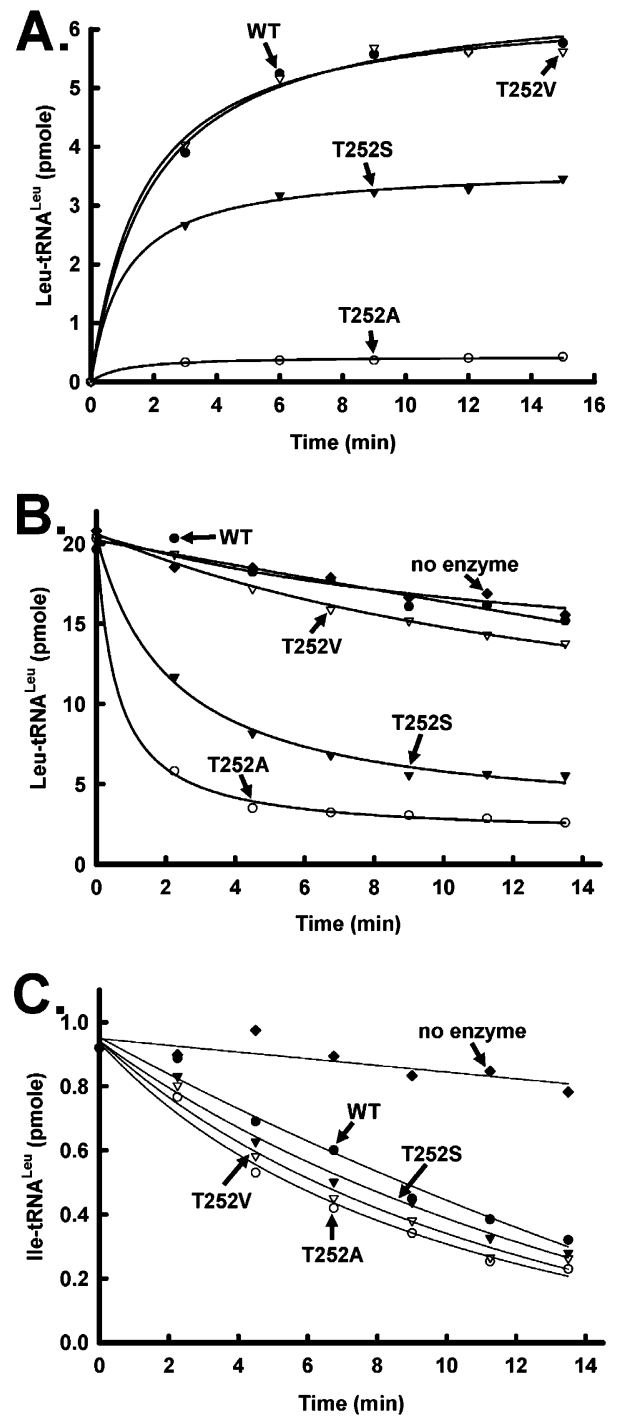

FIGURE 2: Biochemical analysis of T252 single mutations. (A) Leucine aminoacylation of tRNA ${ }^{\text {Leu }}$ by wild-type and mutant LeuRS enzymes. Reaction conditions include $20 \mu \mathrm{M}$ leucine, $2 \mu \mathrm{M}$ tRNA ${ }^{\text {Leu }}$, and $10 \mathrm{nM}$ enzyme. (B) Hydrolytic posttransfer editing activity of cognate amino acid charged Leu-tRNA ${ }^{\text {Leu }}$ by wild-type and mutant LeuRS enzymes. Reaction conditions include $4 \mu \mathrm{M}$ Leu-tRNA ${ }^{\text {Leu }}$ and $10 \mathrm{nM}$ enzyme. (C) Hydrolytic posttransfer editing activity of noncognate isoleucine mischarged tRNA ${ }^{\text {Leu }}$ by wild-type and mutant LeuRS enzymes. Reaction conditions are similar to panel B, except that 20-fold less charged tRNA (200 nM Ile-tRNA $\left.{ }^{\text {Leu}}\right)$ was used to slow the reaction for the detection of subtle mutation-dependent differences. All assays utilized in vitro transcribed tRNA $\mathrm{UAA}_{\mathrm{UAA}}^{\mathrm{Leu}}$. Symbols represent activity by wild-type and mutant LeuRSs as follows: wild-type (WT), solid circle; T252S, solid inverted triangle; T252A, open circle; T252V, open inverted triangle; and no enzyme control, solid diamond.

cantly less than that of aminoacylation. In another example, the T252M mutant LeuRS hydrolyzes Ile-tRNA ${ }^{\text {Leu }}$ at reduced levels as compared to the wild-type LeuRS but fails to yield mischarged product (18). Notably, although targeting somewhat different substrates, the $k_{\text {cat }}$ for Leu-tRNA ${ }^{\text {Leu }}$ hydrolysis by the T252S mutant LeuRS equals that for T252M mutant LeuRS for hydrolysis of Ile-tRNA ${ }^{\text {Leu }}$ (Table 2). We hypothesize that under more physiological conditions, which are better represented by the aminoacylation assay, the T252S mutant LeuRS also fails to achieve the threshold of fidelity that would be required to completely bar the correctly aminoacylated product from the editing active site. 


\begin{tabular}{|c|c|c|c|c|}
\hline (A) leucine & WT & $\mathrm{T} 252 \mathrm{~A}$ & $\mathrm{~T} 252 \mathrm{~S}$ & $\mathrm{~T} 252 \mathrm{~V}$ \\
\hline$K_{\mathrm{M}}(\mu \mathrm{M})$ & 1.5 & 3.6 & 2.0 & 1.6 \\
\hline$k_{\text {cat }}\left(\mathrm{s}^{-1}\right)$ & 6.1 & 0.8 & 5.1 & 6.2 \\
\hline$k_{\text {cat }} / K_{\mathrm{M}}\left(\right.$ normalized $^{b}$ & 1.0 & 0.05 & 0.6 & 1.0 \\
\hline (B) $\mathrm{tRNA}_{\mathrm{UAA}}^{\mathrm{Leu}}$ & WT & $\mathrm{T} 252 \mathrm{~A}$ & $\mathrm{~T} 252 \mathrm{~S}$ & $\mathrm{~T} 252 \mathrm{~V}$ \\
\hline$K_{\mathrm{M}}(\mu \mathrm{M})$ & 1.3 & 2.8 & 1.7 & 1.5 \\
\hline$k_{\text {cat }}\left(\mathrm{s}^{-1}\right)$ & 6.1 & 1.6 & 6.5 & 6.3 \\
\hline$k_{\text {cat }} / K_{\mathrm{M}}\left(\right.$ normalized $^{b}$ & 1.0 & 0.1 & 0.8 & 0.9 \\
\hline
\end{tabular}

${ }^{a}$ Assays utilize in vitro transcribed tRNA $\mathrm{UAAA}_{\mathrm{UA}}^{\mathrm{Leu}}{ }^{b}$ Normalized to the $k_{\text {cat }} / K_{\mathrm{M}}$ for wild-type LeuRS.

\begin{tabular}{|c|c|c|c|c|}
\hline (A) Leu-tRNA UAA $_{\text {Ueu }}^{\text {Leu }}$ & WT & $\mathrm{T} 252 \mathrm{~A}$ & $\mathrm{~T} 252 \mathrm{~S}$ & $\mathrm{~T} 252 \mathrm{~V}$ \\
\hline$K_{\mathrm{M}}(\mu \mathrm{M})$ & 0.4 & 0.6 & 0.3 & 0.5 \\
\hline$k_{\text {cat }}\left(\mathrm{s}^{-1}\right)$ & 0.08 & 3.3 & 0.5 & 0.2 \\
\hline$k_{\text {cat }} / K_{\mathrm{M}}(\text { normalized })^{b}$ & 0.04 & 1.0 & 0.3 & 0.07 \\
\hline (B) Ile-tRNA $\mathrm{UAA}_{\mathrm{UAA}}^{\mathrm{Leu}}$ & WT & $\mathrm{T} 252 \mathrm{~A}$ & $\mathrm{~T} 252 \mathrm{M}$ & $\mathrm{T} 252 \mathrm{~F}$ \\
\hline$K_{\mathrm{M}}(\mu \mathrm{M})$ & 0.7 & 0.8 & 0.4 & 0.7 \\
\hline$k_{\text {cat }}\left(\mathrm{s}^{-1}\right)$ & 1.4 & 2.0 & 0.5 & 0.1 \\
\hline$k_{\text {cat }} / K_{\mathrm{M}}(\text { normalized })^{b}$ & 0.8 & 1.0 & 0.5 & 0.06 \\
\hline
\end{tabular}

${ }^{a}$ Assays utilize in vitro transcribed tRNA $\mathrm{UAA}_{\mathrm{UA}}^{\mathrm{Leu}}{ }^{b}$ Normalized to the $k_{\text {cat }} / K_{\mathrm{M}}$ for T252A mutant LeuRS. ${ }^{c}$ Values for Ile-tRNA ${ }^{\text {Leu }}$ hydrolysis were calculated previously (18). The units have been corrected from this prior publication (41).

It should be noted, however, that interpretation of the kinetic constants measured for the tRNA synthetases, which rely on multiple, sequential reaction steps, may be much more complicated. For example, the directly measured posttransfer editing turnover rate relies on incubation of the enzyme with a very high effective substrate concentration of charged tRNA in solution that is not physiological $(32-34)$ nor complementary to the aminoacylation assay conditions. In addition, hydrolysis under posttransfer editing assay conditions would be simplified as compared to aminoacylation conditions because the former assay likely lacks the poorly understood charged tRNA translocation event from the aminoacylation site. This is supported by previous work that demonstrates that the isolated CP1 domain from IleRS and ValRS can independently carry out posttransfer editing (16). In contrast, under the aminoacylation assay conditions, the editing active site would be expected to be presented with charged tRNA substrate only as it was produced by the synthetic active site. These subsaturating, but more physiologically relevant, substrate conditions would be expected to cause deviations in kinetic parameters that are currently measured based on Michaelis-Menten conditions and assumptions $(5,35)$. Overall, however, these results clearly demonstrate that mutation of the conserved threonine residue to serine does alter substrate specificity and hydrolyzes the correctly charged leu-tRNA ${ }^{\text {Leu }}$ at levels that are sufficient to lower product yields. Importantly, this explains the evolutionary selection of threonine, and not serine, at the critical 252 amino acid position.

We also measured the posttransfer editing activity of noncognate amino acids mischarged to tRNA ${ }^{\text {Leu }}$. The serine, valine, and alanine substitutions hydrolytically cleaved IletRNA $^{\text {Leu }}$ (Figure 2C) and Met-tRNA ${ }^{\text {Leu }}$ (data not shown) at similar or slightly increased rates as compared to the wildtype LeuRS (Table 2). As would be expected, these mutants
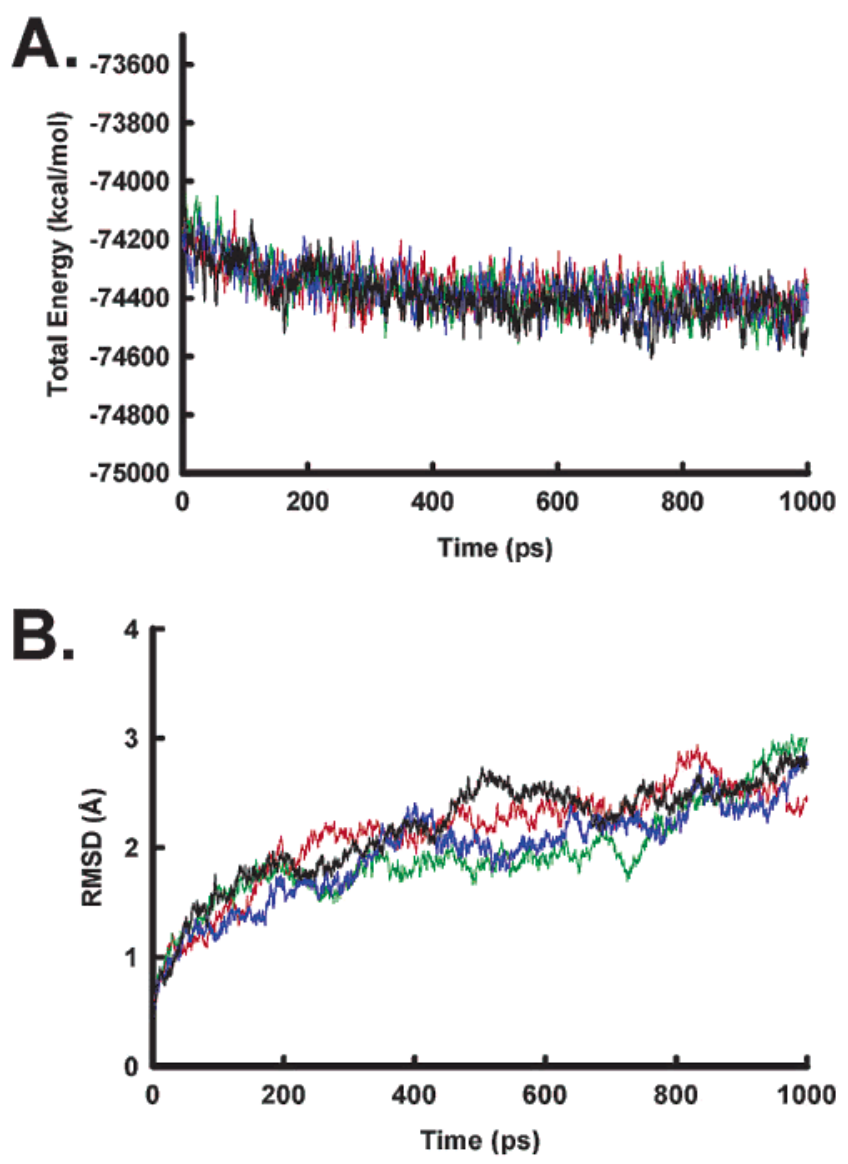

FIGURE 3: MD analyses of the E. coli wild-type and mutant LeuRS CP1 domain models. (A) Total energy throughout the entire $1 \mathrm{~ns}$ simulations. (B) Root-mean-square deviation (RMSD) throughout the entire $1 \mathrm{~ns}$ simulations. The colored lines represent the wildtype or mutant LeuRS CP1 domain models as follows: wild-type, black; T252A, red; T252S, green; and T252V, blue.

could not stably mischarge tRNA ${ }^{\mathrm{Leu}}$ with isoleucine, methionine, or valine (also shown in Figure 8B,D). Thus, homologous mutations at the T252 site primarily affect interactions of the amino acid binding pocket with the cognate leucylated substrate, further suggesting that this position is largely fine-tuned to block the correctly charged Leu-tRNA ${ }^{\text {Leu }}$.

Computational Analysis Indicates Mutation-Dependent Surface Conformational Changes. We employed computational analysis to investigate structural changes imposed by each of the mutations at position 252. The T252A, T252S, and $\mathrm{T} 252 \mathrm{~V}$ mutations were introduced into a homology model (19) of the E. coli LeuRS CP1 domain and further minimized. One nanosecond (ns) MD simulations were performed on the isolated fully hydrated wild-type and mutant LeuRS CP1 domain models. The total energy for each mutant LeuRS CP1 domain model was stable over $1 \mathrm{~ns}$ at $298 \mathrm{~K}$, as was that for the wild-type model (Figure 3A). Likewise, the kinetic and potential energy plots lack any anomalous changes during the simulation time (data not shown). The root-mean-square deviation (RMSD) for the backbone atoms between the first and the subsequent frames in each simulation increased within the first $300 \mathrm{ps}$ and then stabilized to between 2 and $3 \AA$ (Figure 3B). The initial RMSD increase would be expected since the truncated LeuRS CP1 domain model, which was isolated from the main body at residues I224 and L417 to reduce computing time 

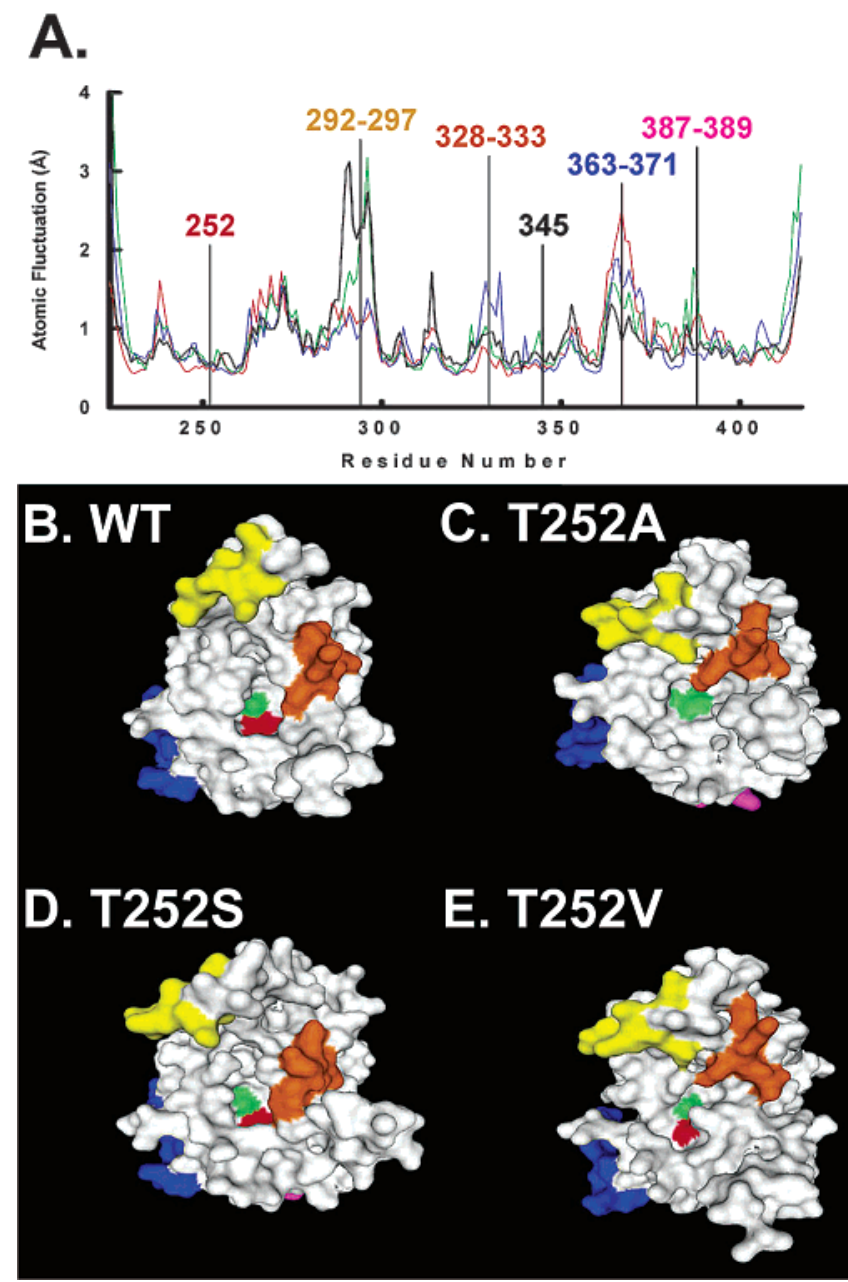

FIGURE 4: MD analyses highlighting the flexible regions of each LeuRS CP1 domain model. The colors of the numbers in panel A correlate to the colored surface residues displayed in panels $\mathrm{B}-\mathrm{E}$. (A) Atomic fluctuations for each of the 194 backbone $C_{\alpha}$ atoms of the wild-type and mutant LeuRS CP1 domain models. The average structures during the final $700 \mathrm{ps}(301-1000 \mathrm{ps})$ of MD were used for this comparison. A solid line and appropriate labels indicate specific residues and regions discussed in the text. The colored lines represent peptide region I224-L417 of the LeuRS CP1 domain model for the following: wild-type, black; T252A, red; T252S, green; and $\mathrm{T} 252 \mathrm{~V}$, blue. (B-E) Solvent accessible surfaces including amino acid side chains for each of the wild-type and mutant LeuRS CP1 domain models. The averaged structures for the final 200 ps $(801-1000 \mathrm{ps})$ of MD were used to create these surface representations. All four structures are oriented similarly based on the $\mathrm{C}_{\alpha}$ carbons of editing active site residues. Differences are highlighted by changes in the surface loops, the two six-amino acid flexible termini (bottom right corner for each model), and side chain rearrangements. Specific residues and peptide regions are colored as follows: V338, light green; X252, red (obscured by residue V338 in panel C); E292-T297, yellow; M328-G333, orange; A363-S371, blue; and F387-G389, pink (only visible in panels C and D).

during MD simulations (Figure 1B, green), required several hundred picoseconds of equilibration.

The localized amino acid editing active sites of the wildtype and mutant CP1 domains were directly compared using the average structures from the final 200 ps of the MD simulations $(801-1000 \mathrm{ps})$. Introduction of each mutation resulted in minimal atomic fluctuations at the critical residue at position 252 (Figure 4A). A second key residue, a universally conserved aspartic acid (D345) that anchors the amino moiety of the amino acid substrate for editing (20,
36), was also stable. As discussed in detail below, local active site geometries of the T252S and T252V mutant LeuRSs most resemble those of the wild-type and T252A mutant LeuRSs, respectively. These conformations are representative of the substrate-free state (19) and are likely altered upon substrate binding (Lee and Briggs, unpublished results). Indepth computational analysis (vide infra) highlighted not only mutation-dependent changes that affect the specificity of the amino acid binding pocket within the editing active site but also geometrical changes within the entire editing active site as well as other long-range alterations.

Mutation-dependent atomic fluctuations at the $\mathrm{C}_{\alpha}$ positions are primarily concentrated in four loops on the surface of the CP1 domain (Figure 4). Comparison of these backbone movements of each LeuRS CP1 domain model over the final 700 ps $(301-1000$ ps) suggests that each mutation induced distinct polypeptide loop movements within the CP1 domain. Relative to the wild-type and T252S LeuRS CP1 domain models, the $\mathrm{T} 252 \mathrm{~A}$ and $\mathrm{T} 252 \mathrm{~V}$ mutations appeared to minimize native atomic fluctuations in the 292-297 loop region that is shown in yellow in Figure $4 \mathrm{~A}-\mathrm{E}$. Notably, this loop region was poorly defined in the X-ray crystallographic data for Thermus thermophilus LeuRS also suggesting that it may be highly flexible (11). Mutations within this loop region were also previously reported to alter amino acid editing activity as well as affect tRNA binding properties $(37,38)$. In addition, amino acid docking studies identified this loop region as a putative second amino acid binding pocket (19).

The T252S and T252A LeuRS CP1 domain models also exhibit idiosyncratic atomic fluctuations of loop residues 387-389, as shown in pink in Figure 4A,C,D. Simulation of the $\mathrm{T} 252 \mathrm{~V}$ mutation exhibited a large distinctive loop fluctuation in residues 328-333 (Figure 4A-E, orange). All mutations stimulated fluctuations at residues $363-371$ with T252A having the largest fluctuations (Figure 4A-E, blue). These coordinated mutation-dependent surface loop movements suggest that sensitive long-distance molecular communication mechanisms exist that might be important to structure, editing activity, tRNA binding, and/or translocation.

Figure $4 \mathrm{~B}-\mathrm{E}$ also displays the entrance to the editing active site and amino acid binding pocket for each mutant LeuRS CP1 domain model. The size of the entrance opening for the T252S LeuRS CP1 domain model is similar to that of the wild-type LeuRS CP1 domain model (Figure 4B,D). Although the opening to the T252A LeuRS CP1 domain model is similar in width, differences within the amino acid binding pocket exist. For example, it is difficult to see residue A252 in Figure 4C due to the short length of its side chain and infringement of the side chain of residue V338, as discussed in more detail below. In contrast, the active site opening of the T252V LeuRS CP1 domain model is clearly distorted (Figure 4E) and appears to be less accessible due to altered orientations and interactions of residues, including T248 and V252. It is likely though, and would be consistent with the experimental data, that these regions of the CP1 domain undergo conformational changes upon binding to the editing substrate, which is absent in these computational models.

Characterization of Mutation-Dependent Geometric Alterations of the Editing Active Site. We calculated mutationdependent variations in the local active site geometry of the 
Table 3: Editing Active Site Distance Measurements between Selected Residues ${ }^{a}$

\begin{tabular}{|c|c|c|c|c|c|c|c|}
\hline \multirow[b]{2}{*}{ residues } & \multirow{2}{*}{$\frac{\text { wild-type }}{D}$} & \multicolumn{2}{|c|}{$\mathrm{T} 252 \mathrm{~A}$} & \multicolumn{2}{|c|}{$\mathrm{T} 252 \mathrm{~S}$} & \multicolumn{2}{|c|}{$\mathrm{T} 252 \mathrm{~V}$} \\
\hline & & $\begin{array}{c}D \\
(\AA)\end{array}$ & $\begin{array}{c}\Delta D^{b} \\
(\AA)\end{array}$ & $\begin{array}{c}D \\
(\AA)\end{array}$ & $\begin{array}{c}\Delta D^{b} \\
(\AA)\end{array}$ & $\begin{array}{c}D \\
(\AA)\end{array}$ & $\begin{array}{l}\Delta D^{b} \\
(\AA)\end{array}$ \\
\hline V245-T248 & 9.96 & 9.79 & -0.17 & 10.02 & +0.06 & 10.30 & +0.34 \\
\hline V245-V335 & 6.00 & 4.90 & -1.10 & 6.29 & +0.29 & 4.41 & -1.59 \\
\hline T248-X252 & 9.31 & 9.13 & -0.18 & 9.86 & +0.55 & 7.53 & -1.78 \\
\hline T248-M336 & 11.82 & 9.37 & -2.45 & 11.68 & -0.14 & 9.42 & -2.40 \\
\hline T248-V338 & 12.94 & 9.40 & -3.54 & 14.08 & +1.15 & 9.57 & -3.37 \\
\hline T248-D342 & 11.47 & 12.62 & +1.15 & 13.67 & +2.20 & 9.21 & -2.26 \\
\hline R249-M336 & 11.91 & 9.16 & -2.75 & 11.25 & -0.66 & 10.90 & -1.01 \\
\hline R249-H341 & 10.02 & 10.81 & +0.79 & 11.34 & +1.32 & 11.92 & +1.90 \\
\hline X252-V335 & 12.57 & 10.55 & -2.02 & 11.04 & -1.53 & 11.67 & -0.90 \\
\hline X252-V338 & 6.84 & 5.47 & -1.37 & 7.18 & +0.34 & 6.82 & -0.02 \\
\hline X252-D342 & 9.96 & 12.04 & +2.08 & 10.20 & +0.24 & 9.38 & -0.58 \\
\hline X252-D345 & 11.73 & 11.74 & +0.01 & 13.44 & +1.71 & 12.45 & +0.72 \\
\hline V335-V338 & 9.41 & 8.89 & -0.52 & 9.41 & +0.00 & 9.31 & -0.10 \\
\hline V335-D345 & 9.93 & 11.26 & +1.33 & 10.73 & +0.80 & 10.84 & +0.91 \\
\hline M336-H341 & 11.78 & 12.31 & +0.53 & 10.37 & -1.41 & 11.68 & -0.10 \\
\hline M336-D342 & 11.41 & 12.34 & +0.93 & 10.04 & -1.36 & 10.61 & -0.80 \\
\hline V338-D342 & 8.45 & 7.17 & -1.28 & 6.35 & -2.10 & 5.99 & -2.46 \\
\hline D342-D345 & 5.41 & 5.73 & +0.32 & 5.80 & +0.39 & 5.54 & +0.13 \\
\hline
\end{tabular}

amino acid binding pocket of the editing active site by measuring distance changes in its width and depth. All measurements between amino acids occurred between their backbone $\mathrm{C}_{\alpha}$ atoms unless otherwise noted. The threoninerich region, $\mathrm{T}_{247} \mathrm{TRPDT}_{252}$, lines one wall of the amino acid binding pocket, whereas selected residues along the peptide $\mathrm{V}_{335}$ MAVPGHDQRD ${ }_{345}$ comprise the remainder of the amino acid binding pocket. Five amino acids located near the rim of the amino acid binding pocket (V245, T248, V335, D342, and D345) were chosen to measure alterations of the substrate entrance (Figure 5A-D and Table 3). In addition, three residues (R249, M336, and H341) were selected to monitor the internal pocket width. The depth of the amino acid binding site was also considered based on measurements between positions V245, T248, T252, V338, and D342 (Figure 6A-D, Table 3).

Measurements for the T252A LeuRS CP1 domain model showed that the opening of its amino acid binding pocket increased an average of nearly $1 \AA$ (Figure 5B and Table 3). However, the internal width between residues R249 and M336 decreased over $2 \AA$. The local backbone geometry of the threonine-rich region is maintained similar to the wildtype structure. In addition, depth measurements between residues A252 and T248 remained comparable to the wildtype LeuRS CP1 domain model (Figure 6B and Table 3), which suggests that the loss of side chain bulk largely influences the increased open area between residues 248 and 252. This increase in both width and depth within the T252A mutant LeuRS CP1 domain likely enhances accessibility to facilitate leucine binding in the amino acid binding pocket of the editing active site for hydrolysis (Figure 2B).

Recent cocrystal structure information (20) and our MD analyses support that a second highly conserved residue (V338) is located near the bottom of the amino acid binding pocket adjacent to T252 and may also contribute to substrate discrimination. The distance between the $\mathrm{C}_{\alpha}$ atoms of residues A252 and V338 in the T252A LeuRS CP1 domain model decreased by $1.37 \AA$. In addition, depth measurements

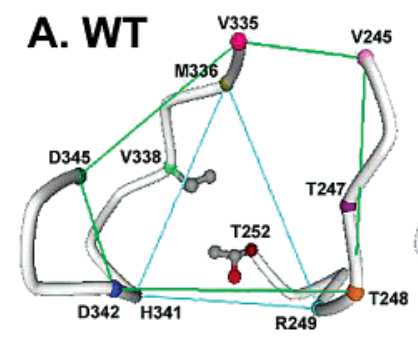

\section{B. T252A}

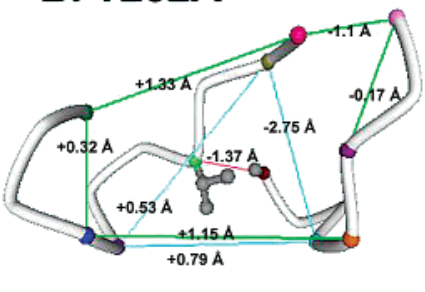

\section{T252S}

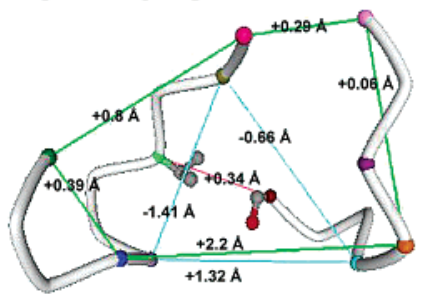

D. T252V

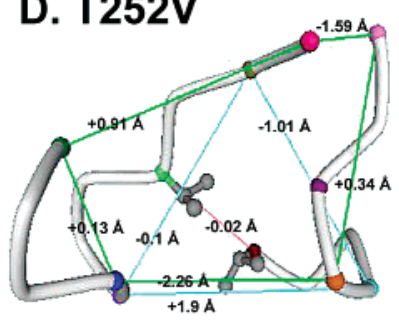

FIGURE 5: Amino acid binding pocket width comparisons between the editing active sites of the wild-type and mutant LeuRS CP1 domain models. (A) Wild-type LeuRS CP1 domain model (WT). (B) T252A mutant LeuRS CP1 domain model. (C) T252S mutant LeuRS CP1 domain model. (D) T252V mutant LeuRS CP1 domain model. A white tube depicts the backbone of peptides V245-T252 and V335-D345. The backbone $\mathrm{C}_{\alpha}$ atoms of selected residues are labeled in panel A and colored as follows: V245, light pink; T247, light purple; T248, orange; R249, light blue; X252, maroon; V335, dark pink; M336, brown; V338, light green; H341, dark purple; D342, blue; and D345, dark green. The side chains of residues X252 and V338 are gray and red to signify their carbon and oxygen substituents, respectively. Surface rim entrance width and internal width distance lines are green and light blue, respectively. A value along each distance line refers to the difference in distance between the mutant and the wild-type LeuRS CP1 domain models (Table 3).

between residues V338 and T248 as well as D342 decreased by 3.54 and $1.28 \AA$, respectively, while residue 252 increased its distance from D342 by $2.08 \AA$. Thus, based on our models, when alanine is substituted at position 252 , the neighboring V338 moves inward and upward within the pocket, and its side chain virtually displaces A252 as it approaches residue T248. This mobility infringes on the relative location of residue A252 in the amino acid binding pocket.

As shown in Figure 4C, A252 cannot be visualized in the surface representation of the amino acid pocket because internal movements of V338 have obscured it. Accordingly, V338 appears to be in an unfavorable position to allow for an amino acid side chain of more than two carbon atoms to bind within the amino acid binding pocket. However, our biochemical data show that leucine, containing two $\mathrm{C}_{\gamma^{-}}$ branched carbon constituents, binds efficiently. Since residues D342 and M336 also move, but in comparable planes to that of V338, while maintaining constant distances between each other, the amino acid binding pocket is extended toward the opposite side of the V338-based wall. Thus, albeit shallower, the extended amino acid binding pocket (represented by distance differences between residues M336 and H341, V335 and D345, as well as A252 and V338 (Figures 5B and 6B and Table 3)) within the T252A mutant LeuRS CP1 domain model would facilitate leucine binding. This is consistent with our biochemical results that show that correctly charged Leu-tRNA ${ }^{\text {Leu }}$ is efficiently hydrolyzed by the T252A mutant LeuRS. 
A. WT

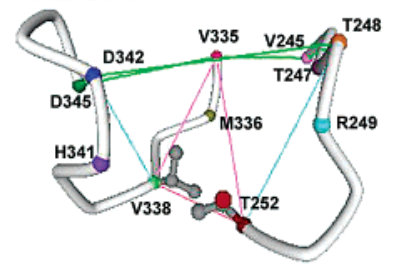

B. T252A
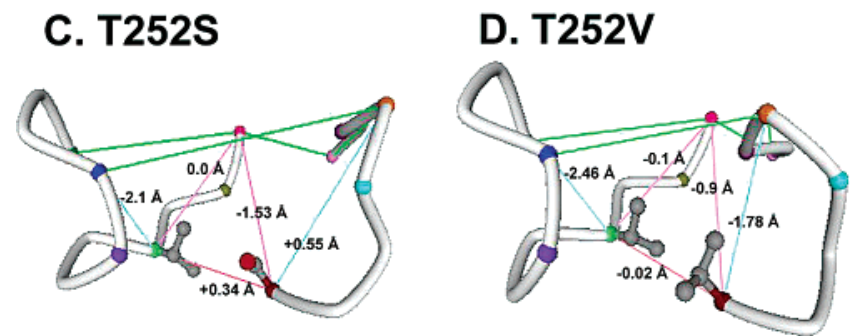

FIGURE 6: Amino acid binding pocket depth comparisons between the editing active sites of the wild-type and mutant LeuRS CP1 domain models. (A) Wild-type LeuRS CP1 domain model (WT). (B) T252A mutant LeuRS CP1 domain model. (C) T252S mutant LeuRS CP1 domain model. (D) T252V mutant LeuRS CP1 domain model. A white tube depicts the backbone of peptides V245-T252 and V335-D345. The backbone $\mathrm{C}_{\alpha}$ atoms of selected residues are labeled in panel A and colored as follows: V245, light pink; T247, light purple; T248, orange; R249, light blue; X252, maroon; V335, dark pink; M336, brown; V338, light green; H341, dark purple; D342, blue; and D345, dark green. The side chains of residues X252 and V338 are gray and red to signify their carbon and oxygen substituents, respectively. Rim width distance lines that are green are maintained from Figure 5 to clarify the relative rotated orientation. Other distance lines are pink, light blue, and orange to represent different areas of the amino acid binding pocket. A value along each distance line refers to the difference in distance between the mutant and the wild-type LeuRS CP1 domain models (Table 3).

The backbone $\mathrm{C}_{\alpha}$ atoms of the T252S LeuRS CP1 domain model are much more similar to the wild-type LeuRS CP1 domain model than that of the T252A LeuRS CP1 domain model (Figures 5C and 6C and Table 3). Similarly, in the T252A LeuRS CP1 domain model, distances between T248 and D342 as well as R249 and H341 indicate that the opening of the amino acid binding pocket increases an average of nearly $1.8 \AA$ and perhaps enhances entry to the binding pocket for the cognate leucine amino acid (Figure 5C and Table 3). However, the distance between residues R249 and M336 within the T252S mutant CP1 domain sustains the wild-type geometry and effectively preserves, at least in part, the internal width of the amino acid binding pocket. Indeed, a decrease in distance between residues M336 and H341 would eliminate an alternative binding position similar to that described above for the T252A LeuRS CP1 domain model. As described below, the amino acid binding position appears to be maintained in the T252S CP1 domain model, relative to the wild-type LeuRS CP1 domain model.

Subtle molecular movements near residue D345 as well as along the threonine-rich peptide result in a depth change. For example, residue D345 increases its distance from 252 by $1.71 \AA$ (Table 3). Likewise, residue T248 increases its distance from 252 by just over $0.5 \AA$ (Figure $6 \mathrm{C}$ and Table 3 ). Collectively, based on the combined computational measurements, we propose that the hydroxyl moiety of $\mathrm{T} 252 \mathrm{~S}$ is required to assist in maintaining the wild-type active site geometry within the amino acid binding pocket. In particular, the hydroxyl moiety helps stabilize and position the side chain of V338 to keep it from infringing on residue 252, as described above for the T252A LeuRS CP1 domain model. Since residues V338 and S252 assume near wildtype positions and the T252S LeuRS hydrolyzes LeutRNA $^{\text {Leu }}$, our data suggest that the reduced discrimination against cognate leucine in the amino acid binding pocket of T252S LeuRS is due to the lack of the bulky methyl moiety of the wild-type $\mathrm{T} 252$ residue.

The T252V mutant LeuRS contains an isosteric side chain that properly excludes leucine from the editing active site as supported by biochemical assays that show it can suitably block Leu-tRNA ${ }^{\text {Leu }}$ from hydrolytic editing (Figure 2B). Our molecular modeling studies suggest, however, that the geometry of the amino acid binding pocket is slightly distorted and appears compressed. For example, distance measurements between the $\mathrm{C}_{\alpha}$ atoms of two residues located at the rim of the pocket (T248 and D342) show a decrease of over $2 \AA$ in the entrance to the amino acid binding pocket relative to the wild-type enzyme (Figure 5D and Table 3).

In contrast, our measurements between neighboring residues, R249 and H341, identified an increase in the internal pocket width greater than that in the T252A and T252S LeuRS CP1 domain models. This larger increase is due primarily to the movements of residues V252 and T248 toward each other, which results in a larger displacement of residue R249, as more clearly visualized in Figure 5D. In addition to the $\mathrm{C}_{\alpha}$ atom, the side chain of R249 is significantly displaced away from the amino acid binding pocket. This side chain movement along with the increase in elevation of residue V252 toward T248 appears to create a shallow, extended binding pocket orientated in an opposite direction to that described previously for the T252A LeuRS CP1 domain model.

On the opposite wall of the amino acid binding pocket in the T252V LeuRS CP1 domain model, a number of residues, including M336, V338, and D342, undergo molecular movements that may create an altered or remodeled amino acid binding pocket. This latter binding pocket is comparable to that found in the T252A LeuRS CP1 domain model discussed previously. Molecular movements within the $\mathrm{T} 252 \mathrm{~V}$ amino acid binding pocket are not as drastic as that observed in the T252A LeuRS CP1 domain model, most likely due to the bulky nature of V252 as compared to A252. This keeps the extended pocket narrower, and as a consequence, blocks leucine from binding. Notably, residue V338 remains consistently positioned in relation to residue V252. The threonine-rich peptide and residue V338 approaches one another decreasing the distance between V338 and T248 by nearly $3.4 \AA$. In addition, the distance in relation to residue D342 decreases by $2.46 \AA$ primarily due to the movement of residue V338. Residue M336 moves along with V338, however, maintaining constant separation. With the large movement of residues T247 and T248, the distance between these two residues and M336 decrease by $2.4 \AA$. Both remodeled amino acid binding pockets appear to be capable of individually binding the noncognate amino acids isoleucine, valine, and methionine for hydrolysis, which is consistent with our biochemical studies. Nevertheless, since Leu-tRNA $^{\text {Leu }}$ hydrolysis remains blocked by the T252V mutant LeuRS, it is likely that either of the compressed mutant amino acid binding pockets undergoes a conforma- 
tional change upon substrate binding (Lee, K. W., and Briggs, J. M., unpublished results).

The geometric similarities between the T252A and T252V as well as the wild-type and T252S LeuRS CP1 domain models suggest that the hydrophobicity of residue 252 may influence the conformation of the amino acid binding pocket. Specifically, we hypothesize that the hydroxyl and methyl moieties of the native T252 residue play important and distinct roles in influencing active site geometry and leucine discrimination, respectively.

Mutation-Dependent Rotations of the 252 Discriminator Side Chain. Changes in the dihedral angles of residue 252 were monitored throughout the MD simulations of the wildtype and T252S LeuRS CP1 domain models to determine the relative location of the hydroxyl moiety of this asymmetric side chain. Dihedral angles were measured for $\mathrm{N}-\mathrm{C}_{\alpha}-\mathrm{C}_{\beta}-\mathrm{O}_{\gamma}$ as diagrammed in Figure 7A. Figure 7B shows that throughout the MD simulation, the dihedral angle for the wild-type hydroxyl moiety exhibited two states. The initial state for the homology model has a dihedral angle near $180^{\circ}$. After the initial $200-300$ ps equilibration period, this hydroxyl moiety rotates to a dihedral angle of near $-50^{\circ}$. Figure 7C shows that the terminal oxygen atom of residue S252 was stable throughout the entire $1 \mathrm{~ns}$ MD simulation in a state analogous to that of the equilibrated wild-type threonine residue. This suggests that the oxygen atoms of each play similar roles in the amino acid binding pocket. As discussed previously, the hydroxyl moiety of residues S252 and T252 may help stabilize not only its own position but also the nearby residue V338 within the amino acid binding pocket. It has been proposed that the hydroxyl moiety might bind a water molecule to sterically hinder leucine from binding in the amino acid binding pocket (21). If this were the case, it is likely that a water molecule would form a hydrogen bond to the mutated S252 residue. However, since our biochemical results demonstrate that the T252S mutant LeuRS could hydrolyze correctly charged Leu-tRNA ${ }^{\text {Leu }}$ (Figure 2B), the additional bulk of the wild-type threonine side chain is clearly an important factor in leucine discrimination.

Structural Synergy within the Amino Acid Binding Pocket. Our computational analyses suggested that the structure and conformation of the amino acid binding pocket is sensitive to substitutions at residue 252 . We combined the T252V substitution with a second mutation, T248A, which also does not greatly affect the editing activity (17). These single and double mutations do not significantly alter leucine-specific aminoacylation (data not shown) or hydrolytic activities (Figure 8A). However, in contrast to each of the single mutants, the double mutant T248A/T252V LeuRS facilitates misaminoacylation of isoleucine (Figure $8 \mathrm{~B}$ ) and significantly decreases Ile-tRNA ${ }^{\text {Leu }}$ hydrolysis (Figure 8C). In addition, low levels of valylation are observed (Figure 8D).

The T252V mutation alone does not appreciably affect the apparent efficiency of LeuRS activity. However, the geometry of the amino acid binding pocket is altered based on our molecular modeling studies. This becomes more biochemically evident when the T248A mutation is introduced in the same local region. The results obtained when combining this mutation with $\mathrm{T} 252 \mathrm{~V}$ suggests that synergistic interactions exist within the amino acid binding pocket of the editing active site. It is also possible that the increased
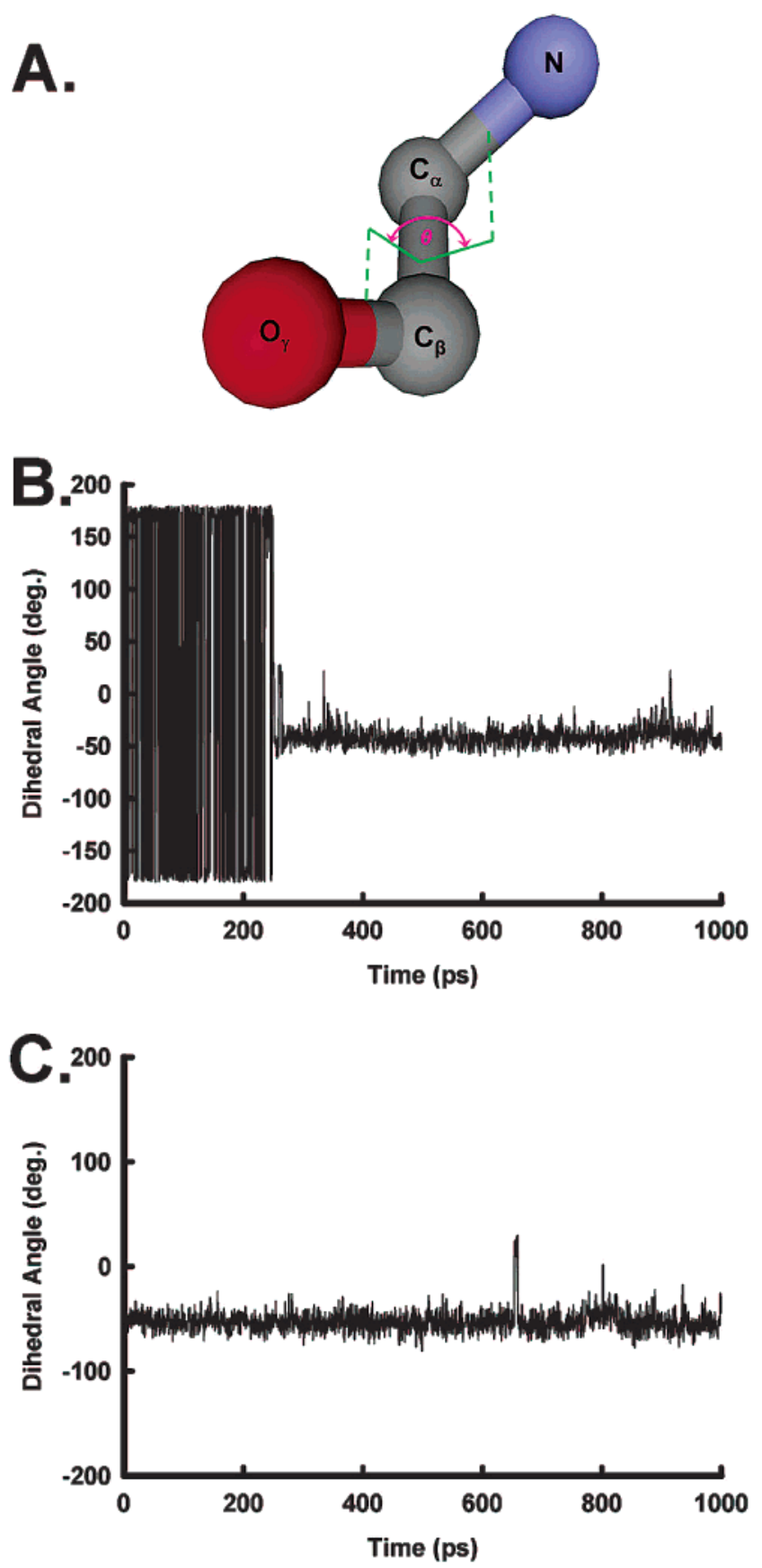

FIGURE 7: Dihedral angle comparison for the side chain terminal oxygen atom of residues T252 and S252. (A) Illustration of a dihedral angle. The dashed green lines are relative bond markers. The solid green lines indicate the angle of rotation to be measured. Atom colors are as follows: nitrogen atom, blue; $\alpha$ - and $\beta$-carbon atoms, gray; and oxygen atom, red. Dihedral angle comparison for the terminal oxygen atoms of residue 252 for the (B) wild-type and (C) T252S mutant LeuRS CP1 domain models. Each angle comparison was constructed from four atoms viewed along the $\mathrm{C}_{\alpha}-$ $\mathrm{C}_{\beta}$ bond axis in the following order: $\mathrm{N}-\mathrm{C}_{\alpha}-\mathrm{C}_{\beta}-\mathrm{O}_{\gamma}$ (as depicted in panel A).

hydrophobicity of the two introduced mutations has further compromised the integrity of the amino acid binding pocket. The X-ray crystal structure of the $T$. thermophilus LeuRS complexed with a posttransfer substrate (PDB code 1OBC) suggests that residue $\mathrm{T} 248$ is involved in a hydrogen bond to the $3^{\prime} \mathrm{OH}$ group of the ribose moiety (20). On the basis of this and previous biochemical data (17) involving the T248A mutant LeuRS, T248 only provides a minor contribu- 

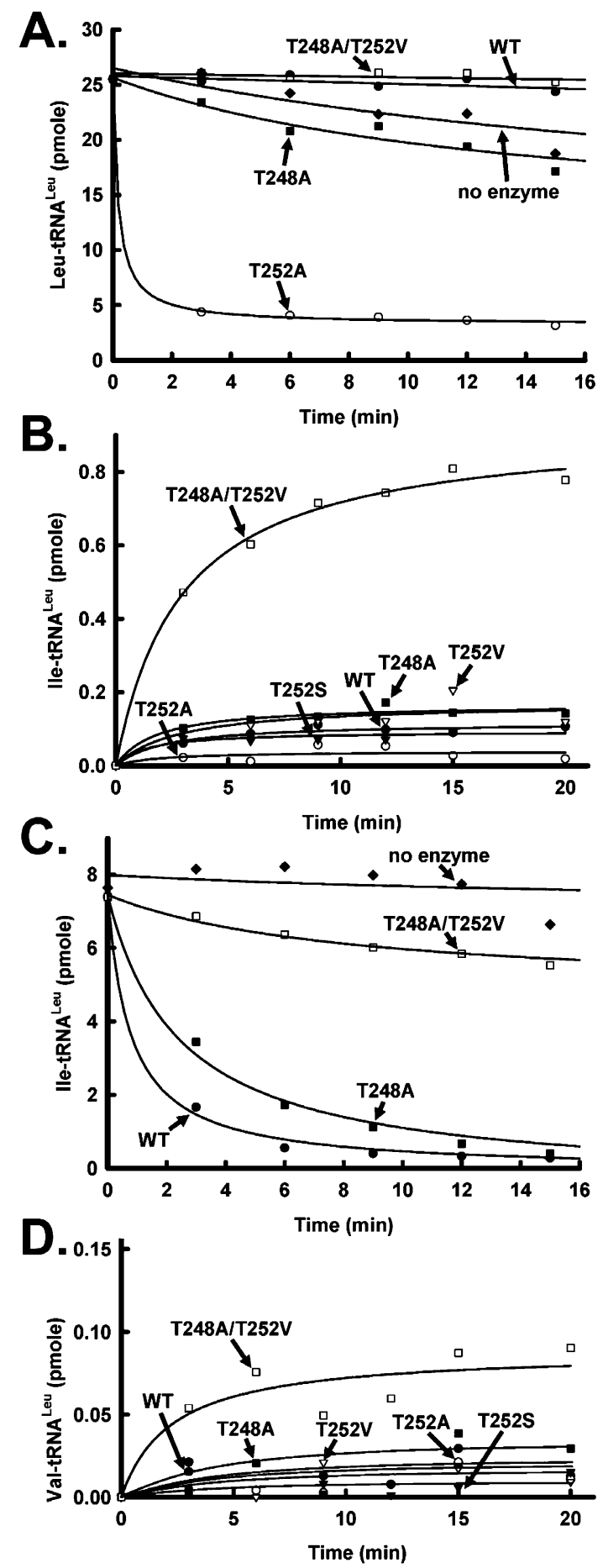

FIGURE 8: Biochemical analysis of wild-type, T248A, and T248A/ T252V mutant LeuRSs. (A) Hydrolytic posttransfer editing activity of cognate leucine charged tRNA ${ }^{\text {Leu }}$ by wild-type and mutant LeuRS enzymes. Reaction conditions include $5 \mu \mathrm{M}$ Leu-tRNA ${ }^{\text {Leu }}$ and 25 $\mathrm{nM}$ enzyme. (B) Isoleucine misaminoacylation of tRNA ${ }^{\mathrm{Leu}}$ by wildtype and mutant LeuRS enzymes. Reaction conditions included 26 $\mu \mathrm{M}$ isoleucine, $5 \mu \mathrm{M}$ tRNA ${ }^{\text {Leu }}$, and $1 \mu \mathrm{M}$ enzyme. (C) Hydrolytic posttransfer editing activity of noncognate isoleucine mischarged to tRNA ${ }^{\text {Leu }}$ by wild-type and mutant LeuRS enzymes. Reaction conditions included $1.5 \mu \mathrm{M}$ Ile-tRNA ${ }^{\mathrm{Leu}}$ and $7.2 \mathrm{nM}$ enzyme. (D) Valine misaminoacylation of tRNA ${ }^{\text {Leu }}$ by wild-type and mutant LeuRS enzymes. Reaction conditions included $26 \mu \mathrm{M}$ valine, $5 \mu \mathrm{M}$ tRNA, and $1 \mu \mathrm{M}$ enzyme. All assays utilized in vitro transcribed tRNA $_{\mathrm{UAA}}^{\mathrm{Leu}}$. Symbols represent enzymatic activity by wild-type and mutant LeuRSs as follows: wild-type (WT), solid circle; T248A, solid square; T248A/T252V, open square; T252V, inverted open triangle; T252S, open circle; and T252A, solid square. tion to editing substrate binding stability in the $E$. coli LeuRS enzyme (Figure 8C). It is possible, however, that upon tampering with the amino acid binding pocket, such as introducing a $\mathrm{T} 252 \mathrm{~V}$ mutation, recognition and discrimination of the editing substrate has become more dependent on the integrity of residue T248.

\section{DISCUSSION}

We previously identified the amino acid binding pocket within the editing active site of LeuRS (17). We also determined that residue T252 acts as a critical discriminator residue by blocking the cognate leucine amino acid from binding efficiently to the editing active site (17). In particular, mutation of the conserved threonine at position 252 to an alanine resulted in a LeuRS mutant enzyme that edits correctly aminoacylated Leu-tRNA ${ }^{\text {Leu }}$.

We combined biochemical experiments and computational analyses to systematically dissect the molecular role of the threonine side chain. Since LeuRS primarily mischarges aliphatic amino acids such as isoleucine, valine, and methionine $(15,18,21)$, one might expect that the amino acid binding pocket would be lined with hydrophobic residues. Therefore, we hypothesized that the highly conserved T252 residue could be substituted by valine without greatly affecting enzymatic activities. However, since the threonine residue is extremely conserved, this also suggests that the hydroxyl moiety is important. The hydroxyl moiety of T252 has been suggested to form an important hydrogen bond with an ordered water molecule $(B$-factor $=44.57)(21)$. If true, then replacement with a serine residue might preserve the hydrogen-bonding properties between residue T252 and the proposed water molecule resulting in wild-type-like enzymatic activity. We tested these hypotheses by introducing a valine and serine mutation for the conserved $\mathrm{T} 252$ residue and investigated their effects on LeuRS editing activity.

Why Not a Serine at the 252 Discriminator Site? As discussed in detail in the Results, much of the geometry of the T252S-based amino acid binding pocket is most similar to the wild-type LeuRS CP1 domain model. However, the amino acid specificity and activity profiles more closely resemble that of the T252A mutant LeuRS. For example, substitution of residue T252 with serine resulted in hydrolytic editing of the cognate Leu-tRNA ${ }^{\text {Leu }}$, similar to the T252A mutant LeuRS. Distance measurements of the opening or entrance to the amino acid binding pocket have increased in both mutant CP1 domain models, which potentially facilitates leucine binding.

Dihedral angle comparisons show that the $\mathrm{O}_{\gamma}$ atom of the $\mathrm{T} 252 \mathrm{~S}$ mutation assumes a similar orientation to that of the wild-type T252 residue. This suggests that the hydroxyl group of T252S LeuRS may effectively mimic the role of that of the T252 side chain in the wild-type LeuRS. However, similar to the T252A substitution, the T252S mutation confers hydrolysis of the correctly charged Leu-tRNA ${ }^{\text {Leu. }}$. Thus, the branched methyl moiety of the highly conserved threonine residue is critical to block the cognate leucine from the editing active site.

Why Not a Valine at the 252 Discriminator Site? In contrast to the serine replacement, the substitution of valine for the conserved T252 residue appeared to be suitable for both aminoacylation and editing activities. Thus, it is curious 
that evolution did not select or interchangeably use valine at the T252 site. However, subsequent computational investigations and mutational analysis suggested that the V252 substitution significantly altered the geometry of the amino acid binding pocket within the editing active site. In particular, at least in the absence of substrate, the T252V substitution appears to compress the opening as well as reduce the depth of the amino acid binding pocket. The valine substitution also results in molecular movements of other residues that alter the shape of the amino acid binding pocket.

The plasticity of the amino acid binding pocket and editing active site (39) of the T252V mutant LeuRS clearly allows efficient editing activity. However, an additional mutation in combination with the valine substitution suggests that the $\mathrm{T} 252 \mathrm{~V}$ mutation did indeed destabilize the integrity of the editing active site. The double mutant LeuRS (T248A/ $\mathrm{T} 252 \mathrm{~V}$ ), in contrast to each of the single mutant LeuRSs (T248A and T252V), impedes Ile-tRNA ${ }^{\text {Leu }}$ hydrolysis resulting in the accumulation of mischarged product. In addition, low levels of valylation of tRNA ${ }^{\text {Leu }}$ are observed.

It is possible that the hydrophilicity of the T252 side chain is important to the conformation of the amino acid binding pocket. The elimination of two polar side chains (via T248A and $\mathrm{T} 252 \mathrm{~V}$ mutations) within the amino acid binding pocket may play a key role in adversely effecting editing activity. It also suggests synergistic or coupled interactions between residue 252 and other local residues, such as at position 248. Synergy between amino acid residues involving the threonine-rich region is also apparent in the editing active site of E. coli IleRS, where a double mutation (T242A/N250A) induced greater editing defects than either single substitution (40). Notably, the analogous residue to E. coli LeuRS T248 is T242 in E. coli IleRS.

Summary. Our results support that both terminal chemical moieties of threonine are important at position 252 to meet the required fidelity of protein synthesis. The bulky methyl moiety maintains selectivity of the amino acid binding pocket that is necessary to block the cognate leucine amino acid. The hydroxyl moiety likely stabilizes its own position as well as that of residue V338 within the amino acid binding pocket. The stabilization by the hydroxyl moiety may be dependent on hydrogen bonding to a water molecule as previously proposed (21). However, this water molecule does not completely bar the leucine amino acid from binding.

MD simulations were performed on each wild-type and mutant LeuRS CP1 domain model to determine structural and editing activity relationships. On the basis of modeling analyses of the four MD trajectories, residue 252 is an important site that helps to define editing active site geometry. For example, introduction of alanine or serine substitutions increased the amino acid binding pocket entrance widths by as much as $2 \AA$, which apparently allows the Leu-tRNA ${ }^{\text {Leu }}$ substrate to enter and subsequently be hydrolyzed. In striking contrast, the $\mathrm{T} 252 \mathrm{~V}$ substitution decreased the pocket entrance by nearly $2 \AA$. Since no apparent enzymatic activity consequence was observed, it is clear that the conformational plasticity of the CP1 domain (39) facilitated editing activity as well as specificity. Because Leu-tRNA ${ }^{\text {Leu }}$ and Ile-tRNA ${ }^{\text {Leu }}$-targeted hydrolysis is facilitated, respectively, by the T252A and T252V mutant LeuRSs, the amino acid binding pocket must undergo conformational changes upon substrate binding (Lee, K. W., and Briggs, J.
M., unpublished results) that would compensate for the apparently distorted binding pockets observed in our ligandfree $\mathrm{CP} 1$ domains during MD analyses.

The combination of somewhat benign mutations greatly decreased hydrolysis activity. These synergistic effects support that the highly conserved T252 and T248 sites are two key structural elements within the editing active site. While the CP1 domain can effectively accommodate one or the other of these individual mutations, substitution of both key stabilization factors dramatically disrupts the integrity of the editing active site, most likely through detrimental geometrical changes.

\section{ACKNOWLEDGMENT}

We thank Dr. Tommie L. Lincecum, Jr. for insightful discussions. We also acknowledge the Institute for Molecular Design at the University of Houston and Accelrys, Inc. for software and the National Resource Allocation Committee for supercomputer time.

\section{REFERENCES}

1. Carter, C. W., Jr. (1993) Annu. Rev. Biochem. 62, 715-48.

2. Martinis, S. A., Plateau, P., Cavarelli, J., and Florentz, C. (1999) Biochimie 81, 683-700.

3. Pauling, L. (1958) The probability of errors in the process of synthesis of protein molecules, in Festschrift für Prof. Dr. Arthur Stöll, pp 597-602, Birkhauser Verlag, Basel, Switzerland.

4. Fersht, A. R., and Kaethner, M. M. (1976) Biochemistry 15, 33426.

5. Fersht, A. R. (1977) Enzyme Structure and Mechanism, W. H. Freeman, San Francisco.

6. Fersht, A. R. (1998) Science 280, 541.

7. Rossmann, M. G., Liljas, A., Brändén, C. I., and Banaszak, L. J. (1975) Evolutionary and structural relationships among dehydrogenases, in The Enzymes (Boyer, P. D., Ed.) 3rd ed., pp 61-102, Academic Press, New York.

8. Moras, D. (1992) Trends Biochem. Sci. 17, 159-64.

9. Hou, Y. M., Shiba, K., Mottes, C., and Schimmel, P. (1991) Proc. Natl. Acad. Sci. U.S.A. 88, 976-80.

10. Starzyk, R. M., Webster, T. A., and Schimmel, P. (1987) Science 237, 1614-8.

11. Cusack, S., Yaremchuk, A., and Tukalo, M. (2000) EMBO J. 19, 2351-61.

12. Fukai, S., Nureki, O., Sekine, S., Shimada, A., Tao, J., Vassylyev, D. G., and Yokoyama, S. (2000) Cell 103, 793-803.

13. Nureki, O., Vassylyev, D. G., Tateno, M., Shimada, A., Nakama, T., Fukai, S., Konno, M., Hendrickson, T. L., Schimmel, P., and Yokoyama, S. (1998) Science 280, 578-82.

14. Silvian, L. F., Wang, J., and Steitz, T. A. (1999) Science 285, 1074-7.

15. Chen, J. F., Guo, N. N., Li, T., Wang, E. D., and Wang, Y. L. (2000) Biochemistry 39, 6726-31.

16. Lin, L., Hale, S. P., and Schimmel, P. (1996) Nature 384, 33-4.

17. Mursinna, R. S., Lincecum, T. L., Jr., and Martinis, S. A. (2001) Biochemistry 40, 5376-81.

18. Mursinna, R. S., and Martinis, S. A. (2002) J. Am. Chem. Soc. $124,7286-7$.

19. Lee, K. W., and Briggs, J. M. (2003) Proteins: Struct., Funct., Genet., in press.

20. Lincecum, T. L., Jr., Tukalo, M., Yaremchuk, A., Mursinna, R. S., Williams, A. M., Sproat, B. S., Van Den Eynde, W., Link, A., Van Calenbergh, S., Grøtli, M., Martinis, S. A., and Cusack, S. (2003) Mol. Cell. 11, 951-63.

21. Tang, Y., and Tirrell, D. A. (2002) Biochemistry 41, 10635-45.

22. Grodberg, J., and Dunn, J. J. (1988) J. Bacteriol. 170, 1245-53.

23. Sampson, J. R., and Uhlenbeck, O. C. (1988) Proc. Natl. Acad. Sci. U.S.A. 85, 1033-7.

24. Martinis, S. A., and Fox, G. E. (1997) Nucleic Acids Symp. Ser. $36,125-8$ 
25. Lincecum, T. L., Jr. (2003) Leucyl-tRNA synthetases: Mechanisms of amino acid activation, aminoacylation, and hydrolytic editing, Ph.D. Thesis, University of Houston, Houston, TX.

26. Schreier, A. A., and Schimmel, P. (1972) Biochemistry 11, 15829.

27. INSIGHTII, v.2000 (2001) Accelrys Inc., San Diego (http:// www.accelrys.com).

28. Brooks, B. R., Bruccoleri, R. E., Olafson, B. D., States, D. J., Swaminathan, S., and Karplus, M. (1983) J. Comp. Chem. 4, 18795.

29. Braun, W., and Go, N. (1985) J. Mol. Biol. 186, 611-26.

30. Kale, L., Skeel, R., Bhandarkar, M., Brunner, R., Gursoy, A., Krawetz, N., Phillips, J., Shinozaki, A., Varadarajan, K., and Schulten, K. (1999) J. Comp. Phys. 151, 283-312.

31. Berendsen, H. J. C., Postma, J. P. M., van Gunsteren, W. F., DiNola, A., and Haak, J. R. (1984) J. Chem. Phys. 81, 3684-90.

32. Negrutskii, B., and Deutscher, M. (1991) Proc. Natl. Acad. Sci. U.S.A. 88, 4991-5.

33. Sang Lee, J., Gyu Park, S., Park, H., Seol, W., Lee, S., and Kim, S. (2002) Biochem. Biophys. Res. Commun. 291, 158-64.
34. Petrushenko, Z. M., Budkevich, T. V., Shalak, V. F., Negrutskii, B. S., and El'skaya, A. V. (2002) Eur. J. Biochem. 269, 4811-8.

35. Fersht, A. R. (1985) Enzyme Structure and Mechanism, Vol. 1, 2nd ed., W. H. Freeman and Co., New York.

36. Bishop, A. C., Nomanbhoy, T. K., and Schimmel, P. (2002) Proc. Natl. Acad. Sci. U.S.A. 99, 585-90.

37. Chen, J. F., Li, T., Wang, E. D., and Wang, Y. L. (2001) Biochemistry 40, 1144-9.

38. Du, X., and Wang, E. D. (2002) Biochemistry 41, 10623-8.

39. Nordin, B. E., and Schimmel, P. (2002) J. Biol. Chem. 277, 20510-7.

40. Hendrickson, T. L., Nomanbhoy, T. K., de Crecy-Lagard, V., Fukai, S., Nureki, O., Yokoyama, S., and Schimmel, P. (2002) Mol. Cell 9, 353-62.

41. Mursinna, R. S., and Martinis, S. A. (2003) J. Am. Chem. Soc. 125,11773

BI034919H 